\section{Hepatoprotection via the IL-6/Stat3 pathway}

\section{Rebecca Taub}

Bristol-Myers Squibb Co., Wallingford, Connecticut, USA

\begin{abstract}
Stat3 is a vital transcription factor that is activated downstream of the gp130 receptor, primarily via IL-6 signaling in adult liver. A new study (see the related article beginning on page 989) demonstrates that Stat3 provides hepatoprotection against Fas-mediated apoptotic liver damage by two mechanisms: direct inactivation of caspases and reduction of reactive oxygen species.
\end{abstract}

J. Clin. Invest. 112:978-980 (2003). doi:10.1172/JCI200319974.
Acute liver failure is caused by a variety of insults, including viral hepatitis, toxic liver damage by poisons or drugs, and ischemia. The liver is the first line of protection against damage by ingested agents, including xenobiotics and drugs. Hepatic injury by these agents frequently results in both hepatic necrosis and apoptosis (1). It is well known that oxidative damage plays a prominent and poison, whereas viral hepatitis and immune-mediated liver damage are believed to occur largely via activation of the Fas apoptotic death pathway. The link between Fas-mediated damage and the induction of reactive oxygen species (ROS) and oxidative damage has only recently been established (2-6).

Growth factors and cytokines, such as HGF and IL-6, promote hepatic survival by stimulating liver regeneration and providing hepatoprotection in a variety of liver-injury models, including Fas-mediated injury, toxic damage caused by hepatotoxins (such as $\mathrm{CCL}_{4}$ ), and ischemic liver injury $(1,7-11)$. These growth factors provide protection against chronic liver injury that

Address correspondence to: Rebecca Taub, Bristol-Myers Squibb Co., PO Box 5100,

5 Research Parkway, Wallingford,

Connecticut 06492, USA.

Phone: (203) 677-6727; Fax: (203) 677-7569;

E-mail: rebecca.taub@bms.com.

Conflict of interest: The author has declared that no conflict of interest exists.

Nonstandard abbreviations used: reactive oxygen species (ROS); signal transducer and activator of transcription-3 (Stat3). role in hepatic injury mediated by drugs ultimately leads to cirrhosis. Part of this protection is mediated by induction of antiapoptotic proteins that regulate the caspase cascade. In this issue of the JCI, Haga and colleagues demonstrate that signal transducer and activator of transcription-3 (Stat3), a key signaling molecule in pathways regulated by IL- 6 and related cytokines, blocks apoptotic injury in two ways: induction of anticaspase regulators; and reduction of oxidative injury via upregulation of an antioxidant protein, Ref-1 (12). These findings provide new insights into common mechanisms of hepatoprotection in both Fasmediated and toxin-mediated acute liver injury and allow predictions about potential therapeutic interventions that could prove beneficial in a variety of liver insults.

\section{The IL-6 signal transduction pathway in liver injury and regeneration}

IL-6 is a critical proregenerative factor and acute-phase inducer in the liver that also confers resistance to liver injury by hepatic toxins, ischemia, and Fas (Figure 1). Its effects are mediated almost exclusively on hepatocytes within the liver. Although the source of IL- 6 within the liver has not been unequivocally established, studies with bone marrow transplantation provide evidence that hepatic Kupffer cells (liver macrophages) are responsible for production of IL- 6 in response to liposaccharide or TNF (13). Secreted IL- 6 acts on neighboring hepatocytes in a paracrine fashion to stimulate liver regeneration and repair. IL- 6 bound to the soluble IL- 6 receptor signals via gp130 and Janus kinase- 1 (JAK-1), leading to activation of the Stat 3 transcription factor and the MAPK signal transduction cascade. IL-6 ${ }^{-/-}$livers induce little Stat 3 in response to IL- 6 activation during liver regeneration after partial hepatectomy, hepatic injury, or acute-phase

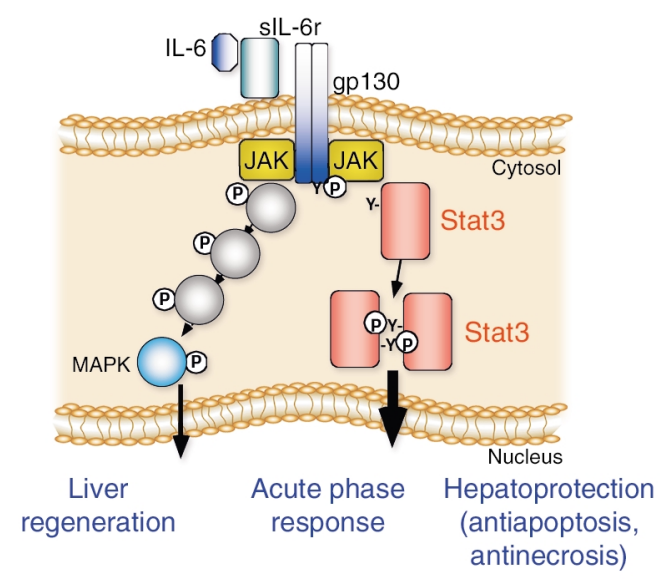

Figure 1

Model for IL-6/Stat3 signaling pathway. IL-6 binds to its soluble receptor, sIL-6r, which binds to the gp130 receptor, resulting in the activation of Janus kinase (JAK). This leads to activation of the MAPK pathway and activation of Stat3 by tyrosine $(Y)$ phosphorylation. Dimerized Stat 3 is able to translocate into the nucleus and activate gene transcription. In the liver, this process promotes liver regeneration, the acute-phase response, and hepatoprotection against Fas and toxic damage. P, phosphate. 
induction, suggesting that Stat 3 may mediate many of the effects of IL-6 (1, 7-10). Conditional Stat3 knockout mice have been used to show that Stat 3 is an important component of the IL-6 response during liver regeneration and the acute-phase response, but Stat 3 does not account for all of the effects of IL-6, particularly those that are mediated by MAPK activation (14). Up to $40 \%$ of the immediate-early genes induced during liver regeneration are regulated at least in part by IL-6, and a significant subset of these are also regulated by Stat3.

\section{Modulation of Stat3 levels in liver cells points to its critical role in hepatocyte survival}

Stat 3 is a vital, ubiquitously expressed protein that is activated by a number of ligands in addition to IL-6 $(15,16)$. It has important roles in mitogenesis and antiapoptosis. Stat 3 has been shown to be involved in the transcriptional upregulation of many genes, not only acting by direct DNA binding, but acting in some cases as a coactivator of transcription factors such as activator protein-1 and hepatocyte nuclear factor-1 (17). Stat3 knockout results in early embryonic lethality, but conditional knockouts provide useful tools to examine the actions of Stat 3 in specific tissues. In the study by Haga et al. (12), two animal models were used to examine the effects of Stat 3 modulation in Fas-mediated liver injury: mice injected with adenoviruses expressing constitutively active Stat 3 and other proteins; and mice with hepatocytespecific Stat3 gene deletions. Adenoviruses injected intravenously normally home to the liver, infecting more than $80 \%$ of hepatocytes and allowing for expression of encoded proteins. Haga et al. demonstrate that constitutively active Stat 3 provided protection against Fas-mediated liver injury, and that Stat3 deficiency led to Fas sensitivity. The antiapoptotic proteins FLIP, $\mathrm{Bcl}-2$, and Bcl-xL, which block caspase activation, are elevated in IL-6-treated livers (9). Haga and colleagues report here that these proteins were also elevated in Stat3-overexpressing livers, providing evidence that $\mathrm{Stat} 3$ mediates the major antiapoptotic effects of IL-6 (Figure 2). Whereas IL-6-mediated ele-

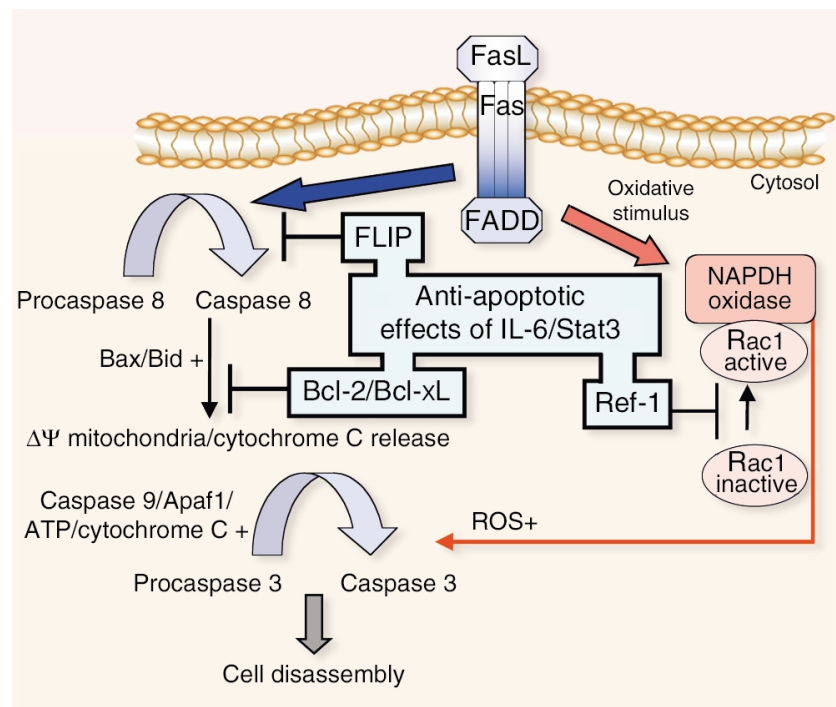

Figure 2

Proposed model for the actions of IL- 6 and Stat 3 that result in hepatoprotection against Fas activation. Interaction of Fas $L$ with its receptor activates the caspase cascade that is blocked by IL- 6 and Stat 3 through the upregulation of FLIP, Bcl-2, and $\mathrm{Bcl}-\mathrm{xL}$. Fas activation also generates an oxidative stimulus that is blocked by the upregulation of Ref- 1 . FADD, Fas-associated death domain; Rac1, RAS-related C3 botulinum toxin substrate 1; Apaf1, apoptotic protease activating factor; Bax, $\mathrm{Bcl} 2$-associated $\mathrm{X}$ protein; Bid, BH3-interacting domain death agonist.

vation of antiapoptotic proteins is largely posttranscriptional (9), mRNA for these proteins was elevated in the Stat3-overexpressing livers (12). This difference could be due to the massive overexpression of Stat 3 and the fact that adenovirus infection confers a degree of transcriptional induction not seen in normal mice.

This mechanistic evaluation was taken to another level by the demonstration that not only anticaspase agents, but also the antioxidant $N$-acetyl cysteine, were able to provide some protection against the effect of Stat 3 deficiency on Fas-mediated apoptosis (12). Having identified ROS as a component of Fas-mediated liver injury, Haga et al. identified an endogenous antioxidant, Ref-1, as a target of Stat3. Expression of Ref-1 provided hepatoprotection, strongly suggesting that Ref- 1 is a critical component of Stat3-mediated hepatoprotection. Ref-1, a dual-function protein upregulated by increases in ROS, is an endonuclease in the base excision repair pathway and a reducing agent that facilitates the DNA-binding properties of redox-sensitive transcription factors (18-21). Ref- 1 is able to suppress ROS generation and hepatic apoptosis (Figure 2).

\section{Hepatoprotection by redox-dependent and -independent mechanisms}

These findings provide important insights into the hepatoprotective properties of IL-6 and its major antiinjury mediator Stat3 (12). Though not yet shown, it is expected that IL-6 induces Ref- 1 , as IL- 6 is the major regulator of Stat 3 activation in Fas- and toxin-mediated liver injury. Stat 3 has not yet been shown to be hepatoprotective in toxic liver damage, but based on these findings, Stat 3 is predicted to be hepatoprotective in liver injury. Toxinmediated liver injury occurs largely through the generation of ROS and direct mitochondrial damage, leading to hepatic necrosis with a lesser degree of apoptosis (1). The level of oxidants may be so high that glutathione is depleted, thereby precluding the activation of caspases, a glutathione-dependent process. By inducing both antioxidant Ref- 1 and caspase inhibitors such as Bcl-2, FLIP, and Bcl-xL, IL-6, Stat3, and similar cytokines are hepatoprotective in a broad spectrum of liver injuries mediated by Fas and liver toxins (22). Cytokines such as IL-6 also promote liver regeneration, another component of the hepatoprotective 
mechanism that restores liver mass after necrotic or apoptotic injury has occurred. The link between intracellular signals resulting in mitogenic and antiapoptotic effects of these agents remains to be completely dissected.

1. Kaplowitz, N.K., and DeLeve, L.D. 2003. Druginduced liver disease. Marcel Dekker Inc. New York, New York, USA. 773 pp.

2. Matsumura, H., et al. 2000. Necrotic death pathway in Fas receptor signaling. J. Cell Biol. 151:1247-1256.

3. Pinkoski, M.J., Brunner, T., Green, D.R., and Lin, T. 2000. Fas and Fas ligand in gut and liver. Am.J. Physiol. Gastrointest. Liver Physiol. 278:G354-G366.

4. Kanzler, S., and Galle, P.R. 2000. Apoptosis and the liver. Semin. Cancer Biol. 10:173-184.

5. Suzuki, Y., Ono, Y., and Hirabayashi, Y. 1998. Rapid and specific reactive oxygen species generation via NADPH oxidase activation during Fasmediated apoptosis. FEBS Lett. 425:209-212.

6. Jayanthi, S., Ordonez, S., McCoy, M.T., and Cadet, J.L. 1999. Dual mechanism of Fas-induced cell death in neuroglioma cells: a role for reactive oxygen species. Brain Res. Mol. Brain Res. 72:158-165.

7. Taub, R., Greenbaum, L.E., and Peng, Y. 1999. Transcriptional regulatory signals define cytokine- dependent and independent pathways in liver regeneration. Semin. Liver Dis. 19:117-127.

8. Kovalovich, K., et al. 2000. Increased toxininduced liver injury and fibrosis in interleukin-6 deficient mice. Hepatology. 31:149-159.

9. Kovalovich, K., et al. 2001. Interleukin- 6 protects against Fas-mediated death by establishing a critical level of anti-apoptotic hepatic proteins FLIP, Bcl-2, and Bcl-xL. J. Biol. Chem. 276:26605-26613.

10. Galun, E., Zeira, E., Pappo, O., Peters, M., and Rose-John, S. 2000. Liver regeneration induced by a designer human IL-6/sIL-6R fusion protein reverses severe hepatocellular injury. FASEB J. 14:1979-1987.

11. Ozaki, M., Haga, S., Zhang, H., Irani, K., and Suzuki, S. 2003. Inhibition of hypoxia/reoxygenationinduced oxidative stress in HGF-stimulated antiapoptotic signaling: role of PI3-K and Akt kinase upon rac1. Cell Death Differ. 10:508-515.

12. Haga, S., et al. 2003. Stat3 protects against Fasinduced liver injury by redox-dependent and -independent mechanisms. J. Clin. Invest. 112:989-998. doi:10.1172/JCI200317970.

13. Debonera, F., et al. 2001. Activation of interleukin6/STAT3 and liver regeneration following transplantation. J. Surg. Res. 96:289-295.

14. Li, W., Liang, X., Kellendonk, C., Poli, V., and Taub, R. 2002. STAT3 contributes to the mitogenic response of hepatocytes during liver regeneration. J. Biol. Chem. 277:28411-28417.
15. Levy, D.E., and Lee, C.-K. 2002. What does Stat3 do? J. Clin. Invest. 109:1143-1148. doi:10.1172/ JCI200215650.

16. Hirano, T., Ishihara, K., and Hibi, M. 2000. Roles of STAT3 in mediating the cell growth, differentiation and survival signals relayed through the IL-6 family of cytokine receptors. Oncogene. 19:2548-2556

17. Leu, J.I., Crissey, M.A., Leu, J.P., Ciliberto, G., and Taub, R. 2001. Interleukin-6-induced STAT3 and AP-1 amplify hepatocyte nuclear factor 1-mediated transactivation of hepatic genes, an adaptive response to liver injury. Mol. Cell. Biol. 21:414-424.

18. Ozaki, M., Suzuki, S., and Irani, K. 2002. Redox factor-1/APE suppresses oxidative stress by inhibiting the rac1 GTPase. FASEBJ. 16:889-890.

19. Ozaki, M., et al. 2000. Inhibition of the Rac1 GTPase protects against nonlethal ischemia/reperfusion-induced necrosis and apoptosis in vivo FASEB J. 14:418-429.

20. Grosch, S., Fritz, G., and Kaina, B. 1998. Apurinic endonuclease (Ref-1) is induced in mammalian cells by oxidative stress and involved in clastogenic adaptation. Cancer Res. 58:4410-4416.

21. Nakamura, H., Nakamura, K., and Yodoi, J. 1997. Redox regulation of cellular activation. Annu. Rev. Immunol. 15:351-369.

22. Hecht, N., et al. 2001. Hyper-IL-6 gene therapy reverses fulminant hepatic failure. Mol. Ther 3:683-687.

\section{Selectin and selectin ligand binding: a bittersweet attraction}

\section{Thomas M. Zollner and Khusru Asadullah}

Corporate Research Business Area Dermatology, Schering AG, Berlin, Germany

Inhibition of leukocyte migration into target organs has long been an attractive, though challenging, basis for anti-inflammatory strategies. However, to date, the manipulation of leukocyte rolling along blood vessels has not yielded successful new therapies. An important study (see the related article beginning on page 1008) may now open new avenues in this exciting field of anti-inflammatory therapies by introducing a putative inhibitor of poly- $N$-acetyllactosamine biosynthesis that affects selectin ligand activity and shows efficacy in a rodent skin inflammation model.

J. Clin. Invest. 112:980-983 (2003). doi:10.1172/JCI200319962.

Address correspondence to: Thomas M. Zollner, Corporate Research Business Area Dermatology, Schering AG, Muellerstrasse 178, D-13342 Berlin, Germany.

Phone: 49-30-468-17235;

Fax: 49-30-468-97235;

E-mail: Thomas.Zollner@Schering.de.

Conflict of interest: The authors are employees and stockholders of Schering AG, which is working on developing antiinflammatory compounds for the treatment of skin diseases.

Nonstandard abbreviations used: sialyl Lewis X $\left(\mathrm{sLe}^{\mathrm{X}}\right)$; cutaneous lymphocyteassociated antigen (CLA); $\beta 1$,4-galactosyltransferase (GalT); $\alpha 2,3$-sialyltransferase (ST3GalIV); $\alpha 1,3$-fucosyltransferase (FucT); P-selectin glycoprotein ligand-1 (PSGL-1); contact hypersensitivity (CHS); peracetylated4-fluorinated-D-glucosamine (4-F-GlcNAc).
Tissue-specific localization of T cells is a requirement for immune surveillance in the skin and in addition plays a pivotal role in the pathogenesis of numerous inflammatory skin disorders. Indeed, the evidence that $T$ cells are crucial factors in mediating psoriasis, allergic contact dermatitis, atopic dermatitis, and cutaneous $\mathrm{T}$ cell lymphomas is so strong that these diseases are now considered as $\mathrm{T}$ cell-mediated dermatoses (1). Consequently, insight into mechanisms of $\mathrm{T}$ cell recruitment to the skin (and other target organs) may lead to novel anti-inflammatory therapies, and the subject is therefore of particular interest.

\section{Selectin and selectin ligand interactions mediate leukocyte rolling along the endothelium}

The multistep cascade of $T$ cell migration has been well described (Figure 1), and the molecular basis for $\mathrm{T}$ cell skin homing has been reviewed recently (2). The first steps of $\mathrm{T}$ cell localization to all tissues include leukocyte tethering and rolling along the vessel wall, which is mediated primarily by interactions between selectin and selectin ligand (3). A number of studies have demonstrated the pivotal role of E- and P-selectin for leukocyte rolling as well as their overlapping and mutually compensating functions (4). Therefore, it was not surprising that a neutralizing antibody solely against E-selectin was found to be without beneficial effects in a recent clinical trial (5). Probably for these reasons, the development of a potent, but E-selectin-specific lowmolecular weight antagonist called ESA-2 (6) was stopped. The lesson learned from these findings was that potent and clinically active selectin antagonists have to interfere with at least two of the three selectins $(\mathrm{E}, \mathrm{P}$, and $\mathrm{L}$ ) in order to show in vivo efficacy. Some such antagonists have been reported recently $(7)$. 